\title{
Software for hard decisions: Scientific influence through interactive visualisation
}

\author{
D. G. Peters
}

Dept Environment and Land Management, Tasmania GPO Box 44a, Hobart, TAS 7001, Australia

Phone:+61 (0)0233 2419, Fax: +61 (0)02 333447

e-mail:dp@parks.tas.gov.au

P. K. Robertson

CSIRO Division of Information Technology

GPO Box 664, Canberra, ACT 2601, Australia

Phone:+61 (0)6 216 7087, Fax: +61 (0)6 2167007 ,

e-mail:phil.robertson@dit.csiro.au

R. L. Cordy

Dept Environment and Land Management, Tasmania

GPO Box 44a, Hobart, TAS 7001, Australia

Phone:+61 (0)0233 2419, Fax: +61 (0)02 333447

e-mail:rc@parks.tas.gov.au

\begin{abstract}
This paper attempts to describe the essential computational functionality required for the understanding and management of the environment and suggests the method by which this functionality might be realised. It is proposed that interactive visualisation will play a key role in enabling the expertise of those involved in the formulation and implementation of government environmental policy, through the provision of new representations and manipulations of environmental information, thus facilitating communication across disciplines and organisational levels in a synthesis of expertise. It is argued that task-oriented user-interfaces will enable access to the advanced computational technologies required to achieve interactive visualisation. Emerging approaches to the systematic design of visualisations and user interaction and the benefits of their application in the environmental arena are outlined. Finally, performance requirements and technologies for realising these benefits, are noted.
\end{abstract}

Keywords

environmental policy, interactive visualisation, user modelling, ecological sustainability 


\section{INTRODUCTION}

\section{Advanced interaction in the "environmental" application domain}

Environmental policy is a major social and political issue - globally, regionally and locally. Policies for resolving of conflicts relating to land and resource use and to standards for environmental protection loom large on most political agendas. Ecological sustainability is generally accepted to be a high priority of responsible government. The operationalising of this high level policy is problematical however.

What benefits can emerging technologies bring to application specialists whose work it is to develop or implement environmental policy? Some technologies have the capacity to be strategic in their use; that is, to allow improved approaches to problem solving, modelling or interpretation. Past examples include geographical information systems, remote sensing, and predictive modelling, the evolution of which have allowed changed approaches to the analysis and representation of land use and biogeography. These technologies have failed to put computers on policy makers' desks in spite of a perceived need for effective decision support. Some of their more serious shortcomings are addressed by "advanced interaction" and its associated technologies.

Advanced interaction is interaction with meaningful data objects and model parameters within the normal working contexts of specialists that is steered by visual feedback. An earlier paper (Robertson, 1994) describes the enabling of specialist's expertise by advanced visual interaction. Much of the basis for the ideas reported here is drawn from that paper.

The aim of this paper is to draw attention to the benefits to nature conservation and environmental management of the application of advanced visual interaction. It is argued that computer systems designed to provide advanced interaction will play a key role in enabling the expertise of those involved in the formulation and implementation of government environmental policy - researchers, planners and managers - through the provision of new representations and manipulations of environmental information, thus facilitating communication across disciplines and organisational levels in a synthesis of expertise.

\section{The purpose of advanced interaction}

Unless people can interact with their data, models or hypotheses, it is likely that a significant portion of their expertise is effectively disabled. Learning by observing alone is much less effective than learning when interaction is possible, suggesting that not only can interaction enable expertise but it can also help build expertise.

Visualisations help us to form mental models of data and processes and to communicate our own mental models to others. Mental models are inferred using both the context and the content of visualisations. Interaction can provide the hand-eye coordination that is so crucial to real-world understanding. The process of experimenting by manipulation - adjusting, modifying, or extending - is part of understanding complex concepts and processes. Interaction is also a very effective way of resolving visual ambiguities and inconsistencies in visual representations. Static representations of complex or abstract phenomena are not easily assimilated, but viewers are nevertheless often unable to provide constructive criticism. Manipulation can help to reveal the underlying real world basis of well-designed visualisations. 


\section{Advanced interaction within a systematic framework}

Systematic design of visualisation, and of interaction, is critical. All too often, visualisations are chosen in an ad-hoc way and fail to illustrate the data or model characteristics of interest. Providing appropriate visualisations and interaction modes will depend on an understanding of how the user expects to carry out the task and on well-defined representations of the task and its products.

A visualisation is generally just part of a broader context, for which there may be many visualisations and interaction possibilities. This "working environment" should be tailored to the user's needs - although usually the reverse is true. Technology, and systematic applications of technology, however, are increasingly offering ways to meet these needs and embed interactive visualisations within the working paradigm of application specialists. Abel et al (1992) describe a prototype of an environmental decision support system which achieved a partial realisation of the goals of advanced interaction.

A systematic approach to the incorporation of interactive visualisation into software systems is essential; as is a systematic approach to developing interaction paradigms consistent with specialists' working environments. Some of today's emerging technologies facilitate these.

\section{THE "ENVIRONMENTAL POLICY" APPLICATION}

\section{The nature of policy development}

Well intentioned, high level policy objectives must be operationalised - reformulated as sets of practical, lower level policies - to have real consequences. Concepts such as biodiversity, ecological sustainability, wilderness, old growth (forests), comprehensive, representative and adequate reservation - the terms in which high level policy is expressed - are complex, abstract and ill-defined. Their assimilation into community mores and the preparedness of industry and government to accommodate them depend on how well the policies they generate reflect credible representations and models of the real world.

\section{The challenge for information technology}

Thus environmental policy, on which decisions for land use and environmental protection are based, is a complex, derived product of scientific expertise and perceived public willingness to accept "tough" decisions. For computers to enable policy makers' expertise, they must facilitate the translation of high level conservation and management objectives into real world terms - they must enable the realisation of scientific expertise and its dissemination into practical policy.

Visualisation is already regarded as essential to the development and communication of complex scientific concepts. That interactive visualisation has the potential to multiply these benefits is obvious. The development of systems and interfaces to realise these benefits is an inevitable consequence of our awareness of the importance of these benefits.

A major part of the policy specialist's task is to integrate expertise from many disciplines. Harnessing the power of advanced technologies to integrate information from many sources is a serious research issue (Abel et al, 1994). The difficulty of providing an application environment for this task is much greater than providing it for particular scientific disciplines. 
The problem is compounded by a relative intolerance to poor performance while at the same time, data requirements to support this task can be massive because of its dependence on multiple derived data sets and their metadata as well as on primary data. It is clear that an intelligent rather than a brutal approach is required to meet this challenge - it is all too easy to offer indefinite increments in processing power and upscaling of inappropriate software.

In spite of the scale of the problem, the evolution of a productive computing environment for environmental policy development and decision support is necessary. By focussing on their end-use of scientific expertise, and a well-defined user model of policy specialists, within the framework of advanced interaction, useful technology will evolve rapidly.

\section{Examples of tasks}

Due to the political importance of the "environment", there are many major policy initiatives with overlapping objectives and integration at this level is an additional overhead, exacerbated by the distribution of responsibility for their implementation among several organisations across all levels of government.

The projects listed do not represent the complete spectrum of environmental policy interests, however, they indicate the range of integration problems and some of the abstract and complex concepts which have to be tackled.

- Wilderness protection

- Rare and threatened species recovery

- Old growth forest conservation

- Ecological monitoring

- State of environment reporting

- Reserve system design

- Sensitivity analysis

- Land use planning

- Analysis of biodiversity

The ways in which the application of advanced interaction might facilitate their handling will be discussed below.

\section{INTERACTION NEEDS}

\section{Types of interaction}

Interaction can range from straightforward pointing or selection, through manipulation and adjusting, to coordinated and highly complex operations. Pointing and selection are well handled by current technologies. Manipulation, however, is much less well handled because of the lack of good input metaphors and supporting devices. Even changing geometrical viewpoint, often the easiest way to resolve ambiguities, generally requires experience with the system being used and often has constraints that are difficult to predict in advance (Felger et al, 1992). This immediately contravenes a core tenet of manipulation in the real world: that we can perform it intuitively or preattentively and allow our attentive resources to be concentrated on observing and interpreting the results of manipulation. With existing computing systems a significant part of the manipulation task requires our attentive mechanisms - just working out how to perform the required manipulation with the tools 
provided - distracting from observing and interpreting the result. The more successful interfaces for viewpoint manipulation are those that map the interactors to the controls of a real-world learned metaphor, such as driving or flying in a simulator.

Interacting with model parameters - that is, parameters that are significant to the process being investigated - can be a powerful way of building an understanding of model scope and constraints. The direct feedback of the effects of adjusting a model parameter can provide information about non-linearities or other types of behaviour that can be very difficult to determine from static views of the results of a particular set of parameter values. A simple example is the delineation of wilderness quality. Current models of wilderness quality are based on linear combinations of variables such as "biophysical naturalness" and "remoteness". Interactive adjustment of the scaling or weighting of these would almost certainly lead to refinement and provide scope for improving confidence in the output index.

Interacting with model parameters also enables the testing of hypotheses ("what-if" scenarios) with particular benefit to the realism of simulations of landscape processes, such as the spread of fire or pathogens. Simultaneous interaction with both the model parameters and the environmental parameters within which the model is running would be particularly beneficial. This would, for example, allow a modeller to optimise the response of a pathogen model to ad hoc settings of an intervention model's parameters.

A general problem for environmental policy development is the delineation of geographical regions. Reserve system evaluation is often based on biophysical regionalisation because such regionalisations are expected to represent summary patterns in the natural distribution of all the biota. The regionalisation task involves finding a concensus among many individual species distributions. While it can be thought of as a segmentation problem in multidimensional space and hence amenable to computer vision algorithms, these provide only a partial solution (Netravali et al, 1995). This task requires interactive guiding of parameter selection ("computational steering") - to determine suitable thresholding and filtering.

Many environmental policies involve dealing with multi-criteria objectives and thresholds of individual criteria. Interactively manipulating thresholds or the weightings of individual components of multi-criteria objectives would help to detect arbitrariness and would facilitate the evolution of robust indices and flexible criteria.

\section{Achieving interaction - performance and system considerations}

These functionalities all require the mapping of model parameters to interaction modes, which means that the model computation must be performed within the interaction latency time. For small problems, computation might be of the order of Mflops (million floating point operations per second), but for models of any reasonable complexity, Gflops (thousand-million floating point operations per second) of computation are likely to be needed. Even this computation size is typical only for screen sized data results. Although processor performance increases steadily, achieving the order of increase in performance needed for interactive modelling requires multiple, or very likely massively parallel, processors.

In fact limitations in screen technologies have allowed, fortuitously, a constraint that has remained pretty well constant for over a decade. Because larger images cannot be displayed, the problem size can often be reduced to that required only for the display resolution. Although not all problems can be so reduced, the constraint did open a window for highly optimised graphics workstations that rely on multiple polygon pipe architectures to achieve the required performance. In general polygon-based algorithms do not scale linearly with 
data size. Scalable performance needs algorithms that scale linearly with data size and with the number of processors introduced.

These very high performance requirements are thus most likely to be met by parallel processors using pixel-based algorithms where possible (Vézina et al, 1992a,b). Pixel-based algorithms have the additional benefit that they can maintain sampling information, often critical for sensed data from which deductions are being drawn. Because polygon algorithms introduce artifacts (polygon edges) that must then be removed by antialiasing methods to avoid their impact on the display or on subsequent modelling stages, it can be very difficult to maintain and represent accurate original sampling information (Greenberg, 1991).

It should also be recognised that interactive visualisation is probably only going to be helpful if it is integrated within standard working tools, which can pose performance demands outside the scope of those tools. This means improved systems design is necessary (Treinish et al, 1992; Ribarsky et al, 1992; Abel et al, 1994; Sharrott, 1993).

\section{Enabling human expertise}

If the performance for interactive visualisation can be achieved, there can be significant benefits by exploiting manipulation and model parameter adjustment. The ability to adjust more than one parameter simultaneously, again something we become very adept at in the real world, can make a substantial difference in exploring the nature of a problem and its potential solutions.

Equally powerful is the potential to exploit interactive forward modelling, and observation of its results, to help solve inverse modelling problems. The specialist is effectively drawn into the process of hypothesising and refining, and in so doing, drawing on experience and learning capacity.

Humans also have an ability to recognise structure or patterns in time varying imagery that may not be evident in static images. Increasing the ability to correlate such patterns with interactively adjusted parameters should more effectively enable analysis skills such as detecting artifacts and surmising their cause, or more clearly distinguishing artifacts from real characteristics.

The environmental policy specialist, who must be able to draw together a variety of complex and incompletely formulated models from many scientific disciplines, is among those who stand to benefit most from interaction.

\section{DESIGNING INTERACTIVE VISUALISATIONS}

Expertise will only be enabled if the data or processes are presented in appropriate ways that avoid or self-resolve ambiguity, are sufficiently consistent for reproducible results, but are sufficiently flexible to allow exploratory work. This suggests developing systematic approaches to determining the visualisations and interactions that are necessary or desirable, and what visualisations and interactions may be undesirable or introduce ambiguity. Often such approaches are based on a clear understanding, or reference model, of the underlying processes of visualisation and interaction, and the resulting assumptions about the user (Haber, 1990, Robertson et al, 1994). 


\section{Choosing visual representations}

There are many different alternative visualisations for data, or combinations of data variables, ranging from various ways of using colour to structural representations (Peters (1991) gives some examples of useful ecological visualisations). The question is which is best for a given set of data, interpretation task, and domain of application.

What is needed first is an understanding, in generic perceptual terms, of what characteristics of a data variable are portrayed by any particular representation that is used to convey the information to an observer. This means determining the information conveying capabilities of the various representations used. Robertson (1991) proposes a method for doing this based on visualisations that are depictions of plausible real-world objects or scenes, the so called natural scene paradigm. This paradigm is based on recognising that we are experienced at abstracting and thus understanding intuitively the properties of real scenes, and that this is the same requirement as we have for individual data variables represented within a visualisation - the ability to abstract variables while also looking at their correlation. So for example, an overhead relief-shaded surface representation of data makes evident the local attributes of a particular value (such as gradients), and the global structure (such as undulations), but not necessarily values at a point because there is no absolute reference for surface height. On the other hand, we can introduce an interactive dipstick to give a reading of surface height at any point, or interactively tip up the surface for a perspective view and improve the ability to estimate the height and thus data value at any point.

The natural scene paradigm is particularly suited to the display of environmental information and not surprisingly, draped perspective views are common products of geographic information systems. The natural scene (with its generic point, local and global attributes) need not represent real world terrain. Peters (1991) proposes that "digital attribute models", which are the generic analogues of digital elevation models, should be exploited by biogeographers. They provide a basis for systematically describing attributes of a data variable that can be made evident in any given representation. And in each case, if the data type represented by the scene property, is known, a formalised description of the representation can be made (Robertson, 1991). Examples are environmental scalars such as mean annual solar radiation and precipitation, biodiversity indices such as species richness and habitat quality, and wilderness quality or any of its components.

\section{Understanding the interpretation task}

Interpretation tasks are generally couched in domain-specific terms that often have a particular contextual meaning. But that does not preclude the specialist from making the bridge to generic terms such as those used to describe the point, local and global attributes of a data variable that a representation can convey. Usually a specialist has sufficient idea of what is being sought to describe it in generic terms. If the data type is also known, this then provides a formalised description of the data and interpretation task - an important feature for official policy implementation.

\section{Matching the representation to the interpretation}

If the representation capability and the interpretation task are both described in generic terms, designing the visualisation and interaction is reduced to a process of matching the 
representation capabilities to the task requirements, and thus steering the choice of representation. This type of systematic approach to design need not be complex to generate consistency and better user feedback than is achieved without it. Neither does it have to be formalised and implemented in software - it can be a set of guiding steps for realising such representations, with the information about representation capability built up through experience.

The need to develop such systematic approaches to design is being increasingly realised in the area of intelligent information presentation systems: unless there is some systematic base rationale for how intelligence or guidance is applied, it is very difficult to apply it usefully. What is increasingly clear is that with improvements in functionality and performance of visualisation systems, the need for designing visualisations is becoming more evident. The emphasis is very much on moving from pretty pictures to informative pictures, where informative implies that the information can be unambiguously decoded, which in turn implies that the encoding approach must be based on systematic design methodologies.

\section{Visualisations for policy support}

The above considerations are particularly important if visualisations are to be used to underpin policy. Another important issue for "official visualisations" is that they must be device independent in the sense that they must have a uniform appearance when displayed on different media. Official hard copy versions are required for reference and must look like the images portrayed on monitors. Also, there is the expectation that policy related material will be published electronically and visualisations should therefore be designed to survive compression and display on popular monitors without loss of information. An example is state of environment reporting in Australia. Methods of satisfying these requirements have been developed (by CSIRO and others) although they are not widely used.

\section{INTERFACE AND INTERACTION PARADIGM}

The visualisation itself, and interaction with its parameters, are just part of the overall interface requirements. Computing systems should support the normal working environment of application specialists, allowing them to work on problems in a familiar manner, consistent with their normal working paradigm, and make the underlying tools or technologies transparent to their use. Existing computing environments do not currently achieve this.

\section{Working paradigms}

Not all environmental policy specialists use computers, but most of those that do, work in an environment that can best be described as a "computational systems and tools" paradigm. That is, they struggle with tools, systems, networks, and incompatibilities. Some work under a "database update" paradigm, but database languages are not sufficient for describing complex modelling or comparison operations and are not easy or natural for policy specialists. A "visualisation-centred" paradigm, based on the recognition that pictures often provide the most effective and influential form of communication, both in static and dynamic form, or even in "hot-spot" enquiry mode sympathetic to an object-oriented software approach, can be an improvement. But even this is several steps away from a "workingapplication environment" paradigm. Somewhere in between is the map making paradigm of 
the conventional geographic information system, which is suitable for making maps but difficult to use for anything else

Much closer is a "document or report" paradigm, where the tender of currency is recognised as a document or report for policy advice or positional analysis. An interface that supports such a document as its main component, and where visualisations and other information provide linked access to data and models transparently, can get close to a "working-application environment" paradigm (This paradigm is popular for publishing material on the Internet). But in the end, even this does not properly reflect the actual working environment of policy specialists.

\section{Task analysis and user modelling}

Finding the right paradigm needs a more formalised framework. The framework must support the representation and application of knowledge about any particular domain of application, and about users' needs for that domain. It must also support awareness of tasks to be undertaken. Current trials in this area are focused on knowledge engineering exploiting knowledge representation within a framework sufficiently formalised to capture and maintain relevant information.

A key component is user modelling: that is, modelling and supporting user needs and behaviour patterns, and adaptability to users' evolving needs. Existing software systems, including user interface management systems, focus on systems' functionalities, and what the user needs to do to access them. What is required is the opposite: to concentrate on user needs and wishes, and what the system needs to do to meet those needs and wishes.

\section{Developing an interaction paradigm}

This leads to the central concept of developing an appropriate interaction paradigm. Such paradigms can form the basis for designing the interaction the user will perform, and the visualisations on which it will be performed, for a complex series of tasks associated with any given application (Carroll, 1991;True, 1992; Nardi et al, 1993). An interaction paradigm can be viewed as a high level metaphor, embracing groupings of individual metaphors each of which may only be independently sustainable in a limited context within a broad framework that allows the expectations for interaction to be predicted. Such a paradigm should evolve from modelling the user expectations of the system, based on a task analysis.

\section{SUMMARY}

Technological advances are increasing the computational resources available to application specialists, but often at the expense of increased complexity of use. Current research on designing user-centred interfaces, based on interaction paradigms, offers the potential to change significantly the way in which computing tools are used by focusing on the user and the task, and not on computational tools. The underlying basis of such approaches, drawing on user modelling and knowledge engineering, can provide a systematic framework for developing working environments tailored to suit the needs of any given application field.

Interactive visualisation can enable the expertise of specialists by closing the hand-eye manipulate-view loop and thus allow complex interaction with models and data. This benefit will only be achieved if the visualisations are designed using systematic approaches, again 
underpinned by knowledge engineering. Advanced visualisation also requires sufficient computational performance to provide interaction with complex model parameters. This performance, several orders of magnitude greater than currently available, will be met by parallel processing technologies supported by transparent software interfaces to application environments.

There seems little doubt that computer systems designed to provide advanced interaction will play a key role in enabling the expertise of those involved in the formulation and implementation of government environmental policy - researchers, planners and managers through the provision of new representations and manipulations of environmental information, thus facilitating communication across disciplines and organisational levels in a synthesis of expertise. The technologies of knowledge engineering and software engineering are key to realising this benefit.

\section{ACKNOWLEDGMENTS}

Much of the material for this paper was developed on the basis of a paper presented by P.K. Robertson at Resource Technology '94 in Melbourne, Australia.

The contribution from staff of the CSIRO Division of Information Technology to the ideas behind this paper is acknowledged, as is the influence of discussions with staff of the Tasmanian Parks and Wildlife Service.

\section{REFERENCES}

Abel, D. J., Yap, S. K., Ackland, R., Cameron, M. A., Smith, D. F., Walker, G., (1992) Environmental Decision Support System Project, International Journal of Geographic Information Systems 6, 193-204.

Abel, D. J., Kilby, P. J., Davis, J. R., (1994) The Systems Integration Problem, InternationalJournal of Geographic Information Systems 8, 1-12.

Carroll, J. (1991) (ed), Designing Interaction, Cambridge University Press, New York.

Felger, W. and Schroder, F., (1992) The Visualization Input Pipeline - Enabling Semantic Interaction in Scientific Visualisation, Eurographics'92, proc. Computer Graphics Forum 11(3).

Greenberg D., (1991) More accurate simulations at faster rates, IEEE Computer Graphics \& Applications 11(1) 23-29.

Haber, R. B. and McNabb, D., (1990) Visualization idioms: A conceptual model for scientific visualization systems, in Visualisation in Scientific Computing (ed. G. Neilson \& B. Shriver), IEEE Computer Society Press, 74-92.

Nardi, B. A. and Zarmer C. L., (1993) Beyond Models and Metaphors: Visual Formalisms in User-Interface Design, Journal of Visual Languages and Computing 4, 5-33.

Netravali, A. N and Haskell, B. G. (1995), Digital Pictures, Plenum Press, New York. 
Peters, D., (1991) Cartographic visualisation and generalisation: Representation of ecological data, Proceedings: Resource Technology 90, Second International Symposium on Advanced Technology in Natural Resource Management, 757-765.

Ribarsky, W., Brown, B., Myerson, T., Feldmann, R., Smith, S., Treinish, L., (1992) ObjectOriented, Dataflow Visualization Systems - A Paradigm Shift?, IEEE Visualization'92, proc., 384-388.

Robertson, P. (1991) A methodology for choosing data representations, IEEE Computer Graphics and Applications 11(3), 56-67.

Robertson, P. K. (1993), Interactive Visualisation, Alustralian Pattern Recognition Society DICTA'93 proc, Sydney, 27-34.

Robertson P. K. and De Ferrari L., (1994) Systematic Approaches to Visualisation: Is a Reference Model Needed?, Proc. ONR Workshop on Data Visualisation, Darmstadt, 1993, in Scientific Visualization: Advances and Challenges, Academic, 1994, 287-305.

Robertson, P. K. (1994), Interactive Visualisation: Its role in enabling specialists' expertise, in Proceedings of Resource Technology '94: new opportunities, best practice; Melbourne.

Sharrott M., (1993) Visualisation using AVS and Massively Parallel Systems, AVS Network News 2(3), 5-8.

Treinish L. A., Butler D. M., Senay H., Grinstein G. G. and Bryson S. T., (1992) Grand Challenge Problems in Visualization Software, IEEE Visualization'92, proc, 366-371.

Treu S., (1992) Interface structures: conceptual, logical, and physical patterns applicable to human-computer interaction, Intl. J. Man-Machine Studies 37, 565-593.

Vézina G. and Robertson P. K., (1992) Data-parallel Visualisation Using Multi-dimensional Transformations, IEEE Frontiers'92: Frontiers of Massively Parallel Computation, proc., 230-239.

\section{BIOGRAPHIES}

David Peters directs the Geographic Information Section of the Tasmanian Parks and Wildlife Service in Australia. He holds an Honours degree in Science from the University of Tasmania. Research interests include the incorporation of artificial intelligence tools with GIS to develop empirical landscape models. Current projects include development of methodologies for terrestrial and marine biophysical regionalisation, biodiversity mapping, forest conservation planning and predictive modelling.

He was the first visiting fellow to ERIN, the Australian Government's Environmental Resources Information Network, where he collaborated with CSIRO on the specification of an environmental decision support system. 
Philip Robertson leads the Human Computer Interaction and Visualisation Program at the CSIRO Division of Information Technology in Australia. He holds BEng, and MSc degrees in Electrical Engineering, and a PhD in Computer Science from the Australian National University. His research interests include algorithms and architecture for visualisation of complex data, colour perception and modelling, massively parallel algorithms, and human computer interface. He has been the Principal Investigator for several major projects in the fields involving research and industry groups, and is a member of the editorial board of IEEE Computer Graphics and Applications.

Rosemary Cordy is a researcher in the GIS Section of the Tasmanian Parks and Wildlife Service. She is an experienced public administrator and has provided the strategic direction of the GIS Section since its inception in 1990. She holds a BA from the University of Tasmania and is currently furthering her studies in Behavioural Science at Monash University. 